\title{
Mappings for Special Functions on Cantor Sets and Special Integral Transforms via Local Fractional Operators
}

\author{
Yang Zhao, ${ }^{1,2}$ Dumitru Baleanu, ${ }^{3,4,5}$ Mihaela Cristina Baleanu, ${ }^{6}$ \\ De-Fu Cheng, ${ }^{2}$ and Xiao-Jun Yang ${ }^{7}$ \\ ${ }^{1}$ Electronic and Information Technology Department, Jiangmen Polytechnic, Jiangmen 529090, China \\ ${ }^{2}$ College of Instrumentation \& Electrical Engineering, Jilin University, Changchun 130061, China \\ ${ }^{3}$ Department of Chemical and Materials Engineering, Faculty of Engineering, King Abdulaziz University, \\ P.O. Box 80204, Jeddah 21589, Saudi Arabia \\ ${ }^{4}$ Department of Mathematics and Computer Sciences, Faculty of Arts and Sciences, Cankaya University, 06530 Ankara, Turkey \\ ${ }^{5}$ Institute of Space Sciences, Magurele, 077125 Bucharest, Romania \\ ${ }^{6}$ Mihail Sadoveanu Theoretical High School, District 2, Street Popa Lazar No. 8, 021586 Bucharest, Romania \\ ${ }^{7}$ Department of Mathematics and Mechanics, China University of Mining and Technology, Xuzhou Campus, \\ Xuzhou, Jiangsu 221008, China
}

Correspondence should be addressed to De-Fu Cheng; chengdefu@jlu.edu.cn

Received 27 August 2013; Accepted 24 September 2013

Academic Editor: Ali H. Bhrawy

Copyright (C) 2013 Yang Zhao et al. This is an open access article distributed under the Creative Commons Attribution License, which permits unrestricted use, distribution, and reproduction in any medium, provided the original work is properly cited.

The mappings for some special functions on Cantor sets are investigated. Meanwhile, we apply the local fractional Fourier series, Fourier transforms, and Laplace transforms to solve three local fractional differential equations, and the corresponding nondifferentiable solutions were presented.

\section{Introduction}

Special functions [1] play an important role in mathematical analysis, function analysis physics, and so on. We recall here some very well examples, the Gamma function [2], hypergeometric function [3], Bessel functions [4], Whittaker function [5], G-function [6], q-special functions [7], Fox's $\mathrm{H}$-functions [8], Mittag-Leffler function [9], and Wright's function [10].

The Mittag-Leffler function had successfully been applied to solve the practical problems [11-15]. For example, the Mittag-Leffler-type functions in fractional evolution processes were suggested [15]. Solutions for fractional reactiondiffusion equations via Mittag-Leffler-type functions were discussed [16]. The Mittag-Leffler stability of fractional order nonlinear dynamic systems was presented [17]. Models based on Mittag-Leffler functions for anomalous relaxation in dielectrics were proposed [18]. In [19], the anomalous relaxation via the Mittag-Leffler functions was reported.
The continuous-time finance based on the Mittag-Leffler function was given [20]. In [21], the fractional radial diffusion in a cylinder based on the Mittag-Leffler function was investigated. In [22], the Mittag-Leffler stability theorem for fractional nonlinear systems with delay was considered. The stochastic linear Volterra equations of convolution type based on the Mittag-Leffler function were suggested in [23]

Recently, based on the Mittag-Leffler functions on Cantor sets via the fractal measure, the special integral transforms based on the local fractional calculus theory were suggested in [24]. In this work, some applications for the local fractional calculus theory are studied in [24-36]. The main aim of this paper is to investigate the mappings for special functions on Cantor sets and some applications of special integral transforms to nondifferentiable problems.

The paper is organized as follows. In Section 2, the mappings for special functions on Cantor sets are investigated. In Section 3, the special integral transforms within local fractional calculus and some applications to nondifferentiable 


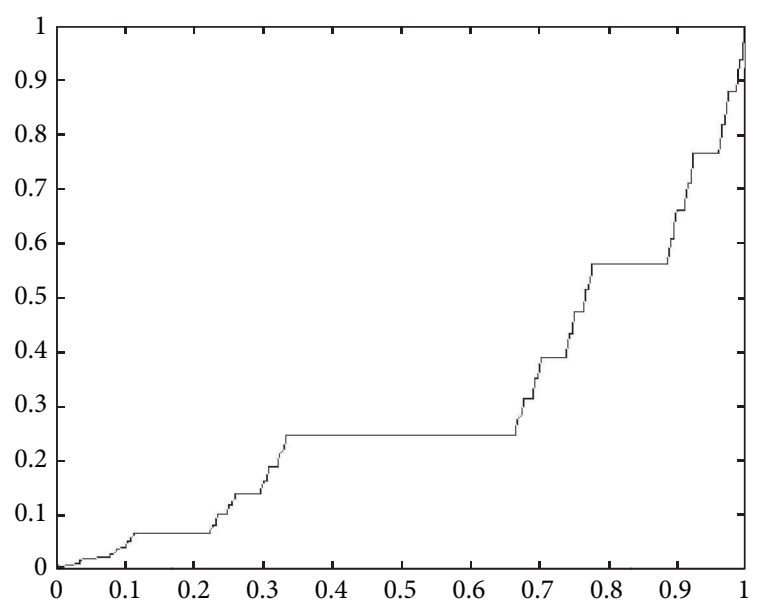

FIGURE 1: Graph of $x^{2 \alpha}$ for $\alpha=\ln 2 / \ln 3$.

problems are presented. Finally, in Section 4, the conclusions are presented.

\section{Mappings for Special Functions on Cantor Sets}

In order to give the mappings for special functions on Cantor sets, we first recall some basic definitions about the fractal measure theory [25].

Let Lebesgue-Cantor staircase function be defined as [25]

$$
H_{\alpha}(F \cap(0, x))=\Gamma(1+\alpha){ }_{0} I_{x}^{(\alpha)} 1,
$$

where $F$ is a cantor set, $H_{\alpha}(\cdot)$ is the $\alpha$-dimensional Hausdorff measure, ${ }_{0} I_{x}^{(\alpha)}(\cdot)$ is local fractional integral operator [24-31], and $\Gamma(\cdot)$ is a Gamma function.

Following (1), we obtain

$$
H_{\alpha}(F \cap(0, x))=x^{\alpha},
$$

which is a Lebesgue-Cantor staircase function. For its graph, please see [28].

In this way, we define some real-valued functions on Cantor sets as follows [24-26].

The Cantor staircase function is defined as [25]

$$
f(x)=x^{2 \alpha},
$$

and its graph is shown in Figure 1.

The Mittag-Leffler functions on Cantor sets are given by $[24,25]$

$$
E_{\alpha}\left(x^{\alpha}\right)=\sum_{k=0}^{\infty} \frac{x^{\alpha k}}{\Gamma(1+k \alpha)}
$$

and we draw the corresponding graph in Figure 2.

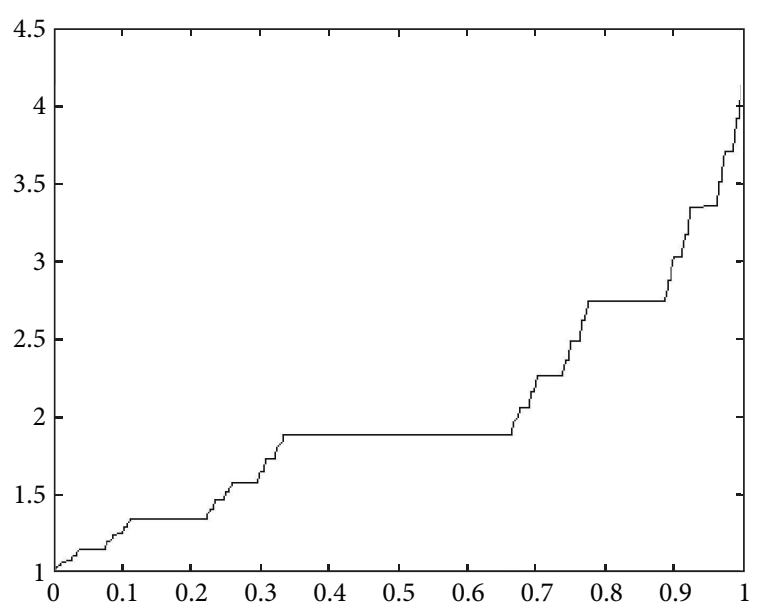

FIGURE 2: Graph of $E_{\alpha}\left(x^{\alpha}\right)$ for $\alpha=\ln 2 / \ln 3$.

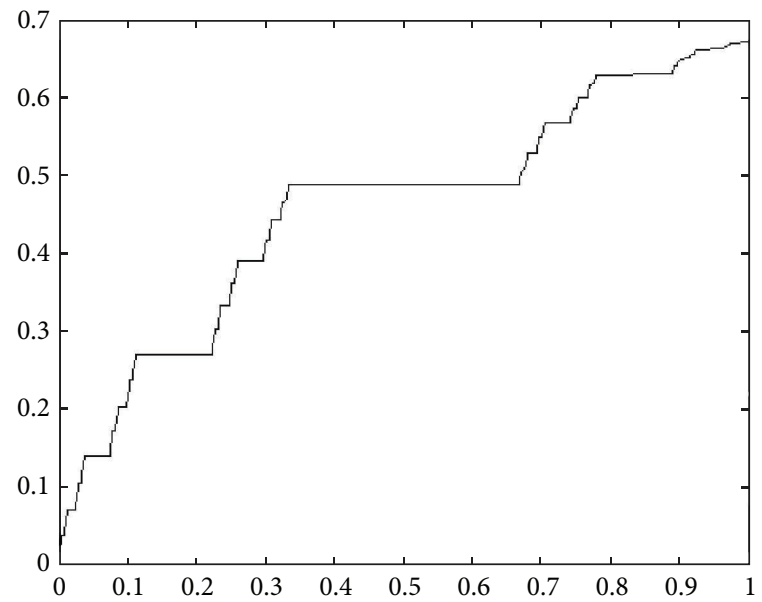

FIgURE 3: Graph of $\sin _{\alpha} x^{\alpha}$ for $\alpha=\ln 2 / \ln 3$.

The sine on Cantor sets is defined by $[24,25]$

$$
\sin _{\alpha} x^{\alpha}=\sum_{k=0}^{\infty}(-1)^{k} \frac{x^{\alpha(2 k+1)}}{\Gamma[1+\alpha(2 k+1)]},
$$

and its corresponding graph is depicted in Figure 3.

The cosine on Cantor sets is [24, 25]

$$
\cos _{\alpha} x^{\alpha}=\sum_{k=0}^{\infty}(-1)^{k} \frac{x^{2 \alpha k}}{\Gamma(1+2 \alpha k)},
$$

with graph in Figure 4.

Hyperbolic sine on Cantor sets is defined by [24, 25]

$$
\sinh _{\alpha} x^{\alpha}=\sum_{k=0}^{\infty} \frac{x^{\alpha(2 k+1)}}{\Gamma[1+\alpha(2 k+1)]},
$$

and we draw its graphs as shown in Figure 5. 


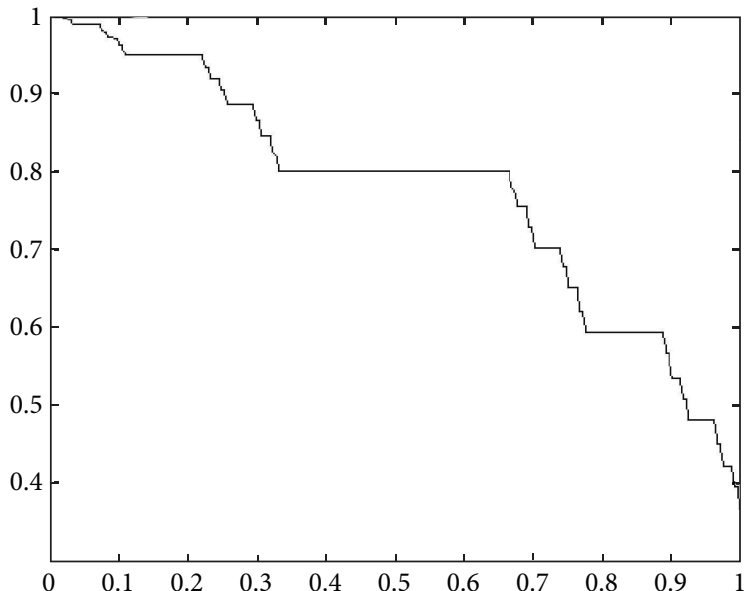

Figure 4: Graph of $\cos _{\alpha} x^{\alpha}$ for $\alpha=\ln 2 / \ln 3$.

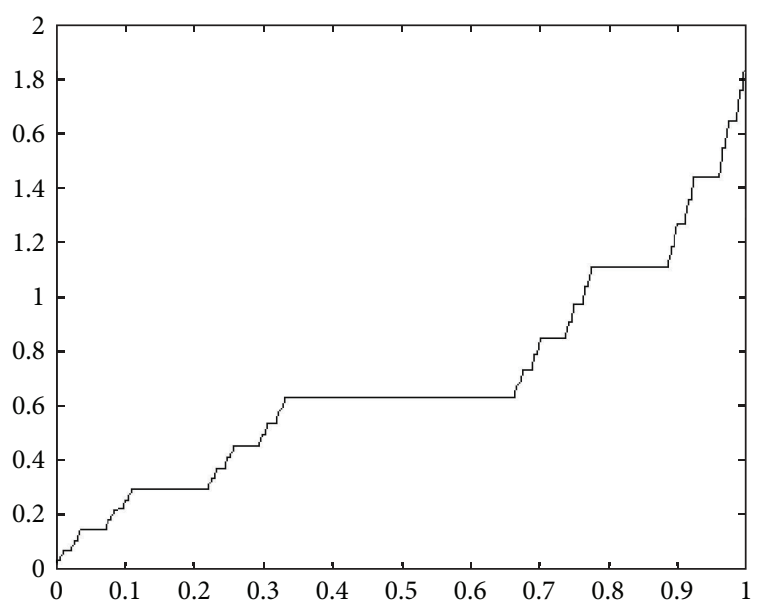

FIGURE 5: Graph of $\sinh _{\alpha} x^{\alpha}$ for $\alpha=\ln 2 / \ln 3$.

Hyperbolic cosine on Cantor sets is defined as [24, 25]

$$
\cosh _{\alpha} x^{\alpha}=\sum_{k=0}^{\infty} \frac{x^{2 \alpha k}}{\Gamma(1+2 \alpha k)},
$$

and its graph is shown in Figure 6.

Following (4)-(8), we have

$$
E_{\alpha}\left(i^{\alpha} x^{\alpha}\right)=\cos _{\alpha} x^{\alpha}+i^{\alpha} \sin _{\alpha} x^{\alpha}
$$

where $i^{\alpha}$ is a fractal unit of an imaginary number [24, 26-32].

If for $\varepsilon, \delta>0$ and $\varepsilon, \delta \in R, f(x)$ satisfies the condition [24-26]

$$
\left|f(x)-f\left(x_{0}\right)\right|<\varepsilon^{\alpha}
$$

for $x \in[a, b]$ we write it as follows:

$$
f(x) \in C_{\alpha}(a, b) .
$$

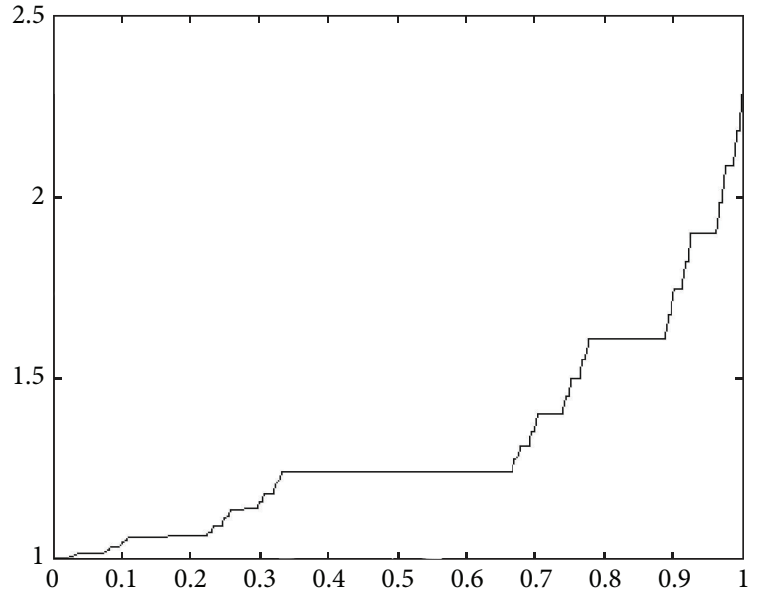

FIgURE 6: Graph of $\cosh _{\alpha} x^{\alpha}$ for $\alpha=\ln 2 / \ln 3$.

\section{Special Integral Transforms within Local Fractional Calculus}

In this section, we introduce the conceptions of special integral transforms within the local fractional calculus concluding the local fractional Fourier series and Fourier and Laplace transforms. After that, we present three illustrative examples.

3.1. Definitions of Special Integral Transforms within Local Fractional Calculus. We here present briefly some results used in the rest of the paper.

Let $f(x) \in C_{\alpha}(-\infty, \infty)$. Local fractional trigonometric Fourier series of $f(x)$ is given by [24, 26-28]

$$
\begin{aligned}
f(x)= & a_{0}+\sum_{i=1}^{\infty} a_{k} \sin _{\alpha}\left(k^{\alpha} \omega_{0}^{\alpha} x^{\alpha}\right) \\
& +\sum_{i=1}^{\infty} b_{k} \cos _{\alpha}\left(k^{\alpha} \omega_{0}^{\alpha} x^{\alpha}\right) .
\end{aligned}
$$

The local fractional Fourier coefficients read as

$$
a_{0}=\frac{1}{T^{\alpha}} \int_{0}^{T} f(x)(d x)^{\alpha},
$$

$$
\begin{aligned}
& a_{k}=\left(\frac{2}{T}\right)^{\alpha} \int_{0}^{T} f(x) \sin _{\alpha}\left(k^{\alpha} \omega_{0}^{\alpha} x^{\alpha}\right)(d x)^{\alpha}, \\
& b_{k}=\left(\frac{2}{T}\right)^{\alpha} \int_{0}^{T} f(x) \cos _{\alpha}\left(k^{\alpha} \omega_{0}^{\alpha} x^{\alpha}\right)(d x)^{\alpha} .
\end{aligned}
$$

We notice that the above results are obtained from Pythagorean theorem in the generalized Hilbert space [24, 26-28].

Let $f(x) \in C_{\alpha}(-\infty, \infty)$. The local fractional Fourier transform of $f(x)$ is suggested by [24, 29-32]

$$
\begin{aligned}
F_{\alpha}\{f(x)\} & =f_{\omega}^{F, \alpha}(\omega) \\
& =\frac{1}{\Gamma(1+\alpha)} \int_{-\infty}^{\infty} E_{\alpha}\left(-i^{\alpha} \omega^{\alpha} x^{\alpha}\right) f(x)(d x)^{\alpha} .
\end{aligned}
$$


The inverse formula is expressed as follows [24, 29-32]:

$$
\begin{aligned}
f(x) & =F_{\alpha}^{-1}\left(f_{\omega}^{F, \alpha}(\omega)\right) \\
& =\frac{1}{(2 \pi)^{\alpha}} \int_{-\infty}^{\infty} E_{\alpha}\left(i^{\alpha} \omega^{\alpha} x^{\alpha}\right) f_{\omega}^{F, \alpha}(\omega)(d \omega)^{\alpha} .
\end{aligned}
$$

Let $f(x) \in C_{\alpha}(-\infty, \infty)$. The local fractional Laplace transform of $f(x)$ is defined as $[24,32,33]$

$$
\begin{aligned}
L_{\alpha}\{f(x)\} & =f_{s}^{L, \alpha}(s) \\
& =\frac{1}{\Gamma(1+\alpha)} \int_{0}^{\infty} E_{\alpha}\left(-s^{\alpha} x^{\alpha}\right) f(x)(d x)^{\alpha} .
\end{aligned}
$$

The inverse formula local fractional Laplace transform of $f(x)$ is derived as $[24,32,33]$

$$
\begin{aligned}
f(x) & =L_{\alpha}^{-1}\left\{f_{s}^{L, \alpha}(s)\right\} \\
& =\frac{1}{(2 \pi)^{\alpha}} \int_{\beta-i \infty}^{\beta+i \infty} E_{\alpha}\left(s^{\alpha} x^{\alpha}\right) f_{s}^{L, \alpha}(s)(d s)^{\alpha},
\end{aligned}
$$

where $f(x)$ is local fractional continuous, $s^{\alpha}=\beta^{\alpha}+i^{\alpha} \infty^{\alpha}$, and $\operatorname{Re}(s)=\beta>0$.

For more details of special integral transforms via local fractional calculus, see $[24,32,33]$ and the references therein.

\subsection{Applications of Local Fractional Fourier Series and Fourier} and Laplace Transforms to the Differential Equation on Cantor Sets. We now present the powerful tool of the methods presented above in three illustrative examples.

Example 1. Let us begin with the local fractional differential equation on Cantor set in the following form:

$$
a \frac{d^{\alpha} y}{d^{\alpha} x}+\text { by }(x)=f(x), \quad x \in(-\infty,+\infty),
$$

where $a$ and $b$ are constants and the nondifferentiable function $f(x)$ is periodic of period $2 \pi$ so that it can be expanded in a local fractional Fourier series as follows:

$$
f(x)=\sum_{n=1}^{\infty} \sin _{\alpha}\left(n^{\alpha} x^{\alpha}\right) .
$$

Here, we give a particular solution in the following form:

$$
\begin{aligned}
y_{p}(x)= & a_{0}+\sum_{n=1}^{\infty} A_{n} \sin _{\alpha}\left(n^{\alpha} x^{\alpha}\right) \\
& +\sum_{n=1}^{\infty} B_{n} \cos _{\alpha}\left(n^{\alpha} x^{\alpha}\right) .
\end{aligned}
$$

Following (20), we have

$$
\begin{aligned}
y_{p}^{(\alpha)}(x)= & \sum_{n=1}^{\infty} A_{n} n^{\alpha} \cos _{\alpha}\left(n^{\alpha} x^{\alpha}\right) \\
& +\sum_{n=1}^{\infty} B_{n} n^{\alpha} \sin _{\alpha}\left(n^{\alpha} x^{\alpha}\right) .
\end{aligned}
$$

Submitting (20)-(21) into (18), we obtain

$$
\begin{aligned}
& a\left(\sum_{n=1}^{\infty} A_{n} n^{\alpha} \cos _{\alpha}\left(n^{\alpha} x^{\alpha}\right)\right. \\
& \left.+\sum_{k=1}^{\infty} B_{n} n^{\alpha} \sin _{\alpha}\left(n^{\alpha} x^{\alpha}\right)\right) \\
& +b\left(a_{0}+\sum_{n=1}^{\infty} A_{n} \sin _{\alpha}\left(n^{\alpha} x^{\alpha}\right)\right. \\
& \left.\quad+\sum_{n=1}^{\infty} B_{n} \cos _{\alpha}\left(n^{\alpha} x^{\alpha}\right)\right) \\
& =\sum_{n=1}^{\infty} \sin _{\alpha}\left(n^{\alpha} x^{\alpha}\right) .
\end{aligned}
$$

Hence, we get

$$
\begin{gathered}
a_{0} b=0, \\
a A_{n} n^{\alpha}+b B_{n}=0, \\
a B_{n} n^{\alpha}+b A_{n}=1 .
\end{gathered}
$$

Therefore, we can calculate

$$
\begin{gathered}
a_{0}=0, \\
A_{n}=-\frac{b}{a^{2} n^{2 \alpha}-b^{2}}, \\
B_{n}=\frac{a n^{\alpha}}{a^{2} n^{2 \alpha}-b^{2}} .
\end{gathered}
$$

In view of (24), we give the solution of (18) as follows:

$$
\begin{aligned}
y_{p}(x)= & -\sum_{n=1}^{\infty} \frac{b}{a^{2} n^{2 \alpha}-b^{2}} \sin _{\alpha}\left(n^{\alpha} x^{\alpha}\right) \\
& +\sum_{n=1}^{\infty} \frac{a n^{\alpha}}{a^{2} n^{2 \alpha}-b^{2}} \cos _{\alpha}\left(n^{\alpha} x^{\alpha}\right) .
\end{aligned}
$$

Example 2. We now consider the following differential equation on Cantor sets:

$$
\frac{d^{2 \alpha} x}{d^{2 \alpha} t}+p x=f(t), \quad+\infty>t>-\infty,
$$

subject to the initial value condition

$$
\left.\frac{d^{\alpha} x}{d^{\alpha} t}\right|_{t=0}=0, \quad x(0)=0,
$$

where $p$ is constant and $f(t)$ is the local fractional continuous function so that its local fractional Fourier transform exists.

Application of local fractional Fourier transform gives

$$
-\omega^{2 \alpha} x_{\omega}^{F, \alpha}(\omega)+p x_{\omega}^{F, \alpha}(\omega)=f_{\omega}^{F, \alpha}(\omega),
$$


so that

$$
\left(-\omega^{2 \alpha}+p\right) x_{\omega}^{F, \alpha}(\omega)=f_{\omega}^{F, \alpha}(\omega)
$$

From (29), we have

$$
x_{\omega}^{F, \alpha}(\omega)=\frac{f_{\omega}^{F, \alpha}(\omega)}{\left(-\omega^{2 \alpha}+p\right)} .
$$

Therefore, taking the inverse formula of local fractional Fourier transform, we have

$$
x(t)=-\frac{p^{-(1 / 2)}}{\Gamma(1+\alpha)} \int_{-\infty}^{t} f(t-\tau) \sin _{\alpha}\left(p^{1 / 2} \tau^{\alpha}\right)(d \tau)^{\alpha} .
$$

Example 3. Let us find the solution to the differential equation on Cantor sets

$$
\frac{d^{2 \alpha} x}{d^{2 \alpha} t}+\frac{d^{\alpha} x}{d^{\alpha} t}-2 x=f(t), \quad t>0
$$

subject to the initial value condition

$$
\left.\frac{d^{\alpha} x}{d^{\alpha} t}\right|_{t=0}=0, \quad x(0)=0
$$

where $f(t)$ is the local fractional continuous function so that its local fractional Laplace transform exists.

Taking the local fractional Laplace transform, from (32), we have

$$
\begin{aligned}
& \left(s^{2 \alpha} x_{s}^{L, \alpha}(s)-s^{\alpha} x(0)-x^{(\alpha)}(0)\right)+\left(s^{\alpha} x_{s}^{L, \alpha}(s)-x(0)\right) \\
& \quad+2 x_{s}^{L, \alpha}(s)=f_{s}^{L, \alpha}(s),
\end{aligned}
$$

so that

$$
x_{s}^{L, \alpha}(s)=\frac{f_{s}^{L, \alpha}(s)}{s^{2 \alpha}+s^{\alpha}-2} .
$$

When the local fractional convolution of two functions is given by [24]

$$
f_{1}(t) * f_{2}(t)=\frac{1}{\Gamma(1+\alpha)} \int_{0}^{t} f_{1}(t-\tau) f_{2}(\tau)(d \tau)^{\alpha}
$$

and the local fractional Laplace transform of $f_{1}(t) * f_{2}(t)$ is [24]

$$
L_{\alpha}\left\{f_{1}(t) * f_{2}(t)\right\}=f_{s, 1}^{L, \alpha}(s) f_{s, 2}^{L, \alpha}(s),
$$

the inverse formula of the local fractional Laplace transform together with the local fractional convolution theorem gives the solution

$$
x(t)=\frac{1}{\Gamma(1+\alpha)} \int_{0}^{t} f(t-\tau)\left(E_{\alpha}\left(-2 \tau^{\alpha}\right)+E_{\alpha}\left(\tau^{\alpha}\right)\right)(d \tau)^{\alpha} .
$$

\section{Conclusions}

In this work, we investigated the mappings for special functions on Cantor sets and special integral transforms via local fractional calculus, namely, the local fractional Fourier series, Fourier transforms, and Laplace transforms, respectively. These transformations were applied successfully to solve three local fractional differential equations, and the nondifferentiable solutions were reported.

\section{References}

[1] G. E. Andrews, R. Askey, and R. Roy, Special Functions, Cambridge University Press, Cambridge, UK, 1999.

[2] Á. Elbert and A. Laforgia, "On some properties of the gamma function," Proceedings of the American Mathematical Society, vol. 128, no. 9, pp. 2667-2673, 2000.

[3] N. Virchenko, S. L. Kalla, and A. Al-Zamel, "Some results on a generalized hypergeometric function," Integral Transforms and Special Functions, vol. 12, no. 1, pp. 89-100, 2001.

[4] J. Choi and P. Agarwal, "Certain unified integrals associated with Bessel functions," Boundary Value Problems, vol. 2013, no. 1, pp. 1-9, 2013.

[5] P. J. McNamara, "Metaplectic Whittaker functions and crystal bases," Duke Mathematical Journal, vol. 156, no. 1, pp. 1-31, 2011.

[6] E. W. Barnes, "The theory of the G-function," Quarterly Journal of Mathematics, vol. 31, pp. 264-314, 1899.

[7] R. Floreanini and L. Vinet, " $U_{q}(s l(2))$ and $q$-special functions," Contemporary Mathematics, vol. 160, p. 85, 1994.

[8] H. M. Srivastava, K. C. Gupta, and S. P. Goyal, The H-Functions of One and Two Variables with Applications, South Asian Publishers, New Delhi, India, 1982.

[9] R. Gorenflo, A. A. Kilbas, and S. V. Rogosin, "On the generalized Mittag-Leffler type functions," Integral Transforms and Special Functions, vol. 7, no. 3-4, pp. 215-224, 1998.

[10] R. K. Saxena and M. Saigo, "Certain properties of fractional calculus operators associated with generalized Mittag-Leffler function," Fractional Calculus \& Applied Analysis, vol. 8, no. 2, pp. 141-154, 2005.

[11] D. Baleanu, K. Diethelm, E. Scalas, and J. J. Trujillo, Fractional Calculus Models and Numerical Methods, Series on Complexity, Nonlinearity and Chaos, World Scientific, Singapore, 2012.

[12] A. A. Kilbas, H. M. Srivastava, and J. J. Trujillo, Theory and Applications of Fractional Differential Equations, vol. 204, Elsevier, Amsterdam, The Netherlands, 2006.

[13] J. Sabatier, O. P. Agrawal, and J. A. T. Machado, Advances in Fractional Calculus: Theoretical Developments and Applications in Physics and Engineering, Springer, New York, NY, USA, 2007.

[14] S. Hu, Y. Q. Chen, and T. S. Qiu, Fractional Processes and Fractional-Order Signal Processing: Techniques and Applications, Springer, New York, NY, USA, 2012.

[15] F. Mainardi and R. Gorenflo, "On Mittag-Leffler-type functions in fractional evolution processes," Journal of Computational and Applied Mathematics, vol. 118, no. 1-2, pp. 283-299, 2000.

[16] R. K. Saxena, A. M. Mathai, and H. J. Haubold, "Fractional reaction-diffusion equations," Astrophysics and Space Science, vol. 305, no. 3, pp. 289-296, 2006.

[17] Y. Li, Y. Chen, and I. Podlubny, "Mittag-Leffler stability of fractional order nonlinear dynamic systems," Automatica, vol. 45, no. 8, pp. 1965-1969, 2009.

[18] E. C. de Oliveira, F. Mainardi, and J. Vaz Jr., "Models based on Mittag-Leffler functions for anomalous relaxation in dielectrics," The European Physical Journal Special Topics, vol. 193, no. 1, pp. 161-171, 2011.

[19] D. S. F. Crothers, D. Holland, Y. P. Kalmykov, and W. T. Coffey, "The role of Mittag-Leffler functions in anomalous relaxation," Journal of Molecular Liquids, vol. 114, no. 1-3, pp. 27-34, 2004.

[20] E. Scalas, R. Gorenflo, and F. Mainardi, "Fractional calculus and continuous-time finance," Physica A, vol. 284, no. 1-4, pp. 376$384,2000$. 
[21] B. N. N. Achar and J. W. Hanneken, "Fractional radial diffusion in a cylinder," Journal of Molecular Liquids, vol. 114, no. 1-3, pp. 147-151, 2004.

[22] S. J. Sadati, D. Baleanu, A. Ranjbar, R. Ghaderi, and T. Abdeljawad, "Mittag-Leffler stability theorem for fractional nonlinear systems with delay," Abstract and Applied Analysis, vol. 2010, Article ID 108651, 7 pages, 2010.

[23] S. W. J. Welch, R. A. L. Rorrer, and R. G. Duren Jr., "Application of time-based fractional calculus methods to viscoelastic creep and stress relaxation of materials," Mechanics Time-Dependent Materials, vol. 3, no. 3, pp. 279-303, 1999.

[24] X. J. Yang, Local Fractional Functional Analysis and Its Applications, Asian Academic Publisher, Hong Kong, China, 2011.

[25] X. J. Yang, Advanced Local Fractional Calculus and Its Applications, World Science, New York, NY, USA, 2012.

[26] M.-S. Hu, R. P. Agarwal, and X.-J. Yang, "Local fractional Fourier series with application to wave equation in fractal vibrating string," Abstract and Applied Analysis, vol. 2012, Article ID 567401, 15 pages, 2012.

[27] Y. Zhang, A. Yang, and X.-J. Yang, "1-D heat conduction in a fractal medium: a solution by the local fractional Fourier series method," Thermal Science, vol. 17, no. 3, pp. 953-956, 2013.

[28] Y.-J. Yang, D. Baleanu, and X.-J. Yang, "Analysis of fractal wave equations by local fractional Fourier series method," Advances in Mathematical Physics, vol. 2013, Article ID 632309, 6 pages, 2013.

[29] X. J. Yang, D. Baleanu, and J. A. T. Machado, "Mathematical aspects of Heisenberg uncertainty principle within local fractional Fourier analysis," Boundary Value Problems, vol. 2013, no. 1, pp. 131-146, 2013.

[30] A. M. Yang, Y. Z. Zhang, and Y. Long, "The Yang-Fourier transforms to heat-conduction in a semi-infinite fractal bar," Thermal Science, vol. 17, no. 3, pp. 707-7713, 2013.

[31] F. Gao, W. P. Zhong, and X. M. Shen, "Applications of YangFourier transform to local fractional equations with local fractional derivative and local fractional integral," Advanced Materials Research, vol. 461, pp. 306-310, 2012.

[32] J.-H. He, "Asymptotic methods for solitary solutions and compactons," Abstract and Applied Analysis, vol. 2012, Article ID 916793, 130 pages, 2012.

[33] C. F. Liu, S. S. Kong, and S. J. Yuan, "Reconstructive schemes for variational iteration method within Yang-Laplace transform with application to fractal heat conduction problem," Thermal Science, vol. 17, no. 3, pp. 715-721, 2013.

[34] G. S. Chen, "Generalizations of hölder's and some related integral inequalities on fractal space," Journal of Function Spaces and Applications, vol. 2013, Article ID 198405, 9 pages, 2013.

[35] A. Carpinteri, B. Chiaia, and P. Cornetti, "The elastic problem for fractal media: basic theory and finite element formulation," Computers and Structures, vol. 82, no. 6, pp. 499-508, 2004.

[36] A. K. Golmankhaneh, V. Fazlollahi, and D. Baleanu, "Newtonian mechanics on fractals subset of real-line," Romania Reports in Physics, vol. 65, pp. 84-93, 2013. 


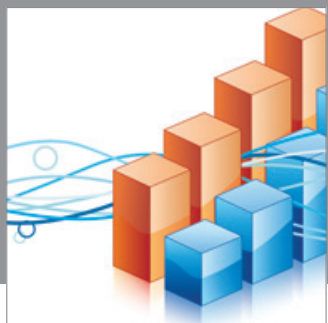

Advances in

Operations Research

mansans

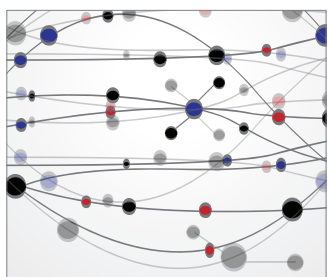

The Scientific World Journal
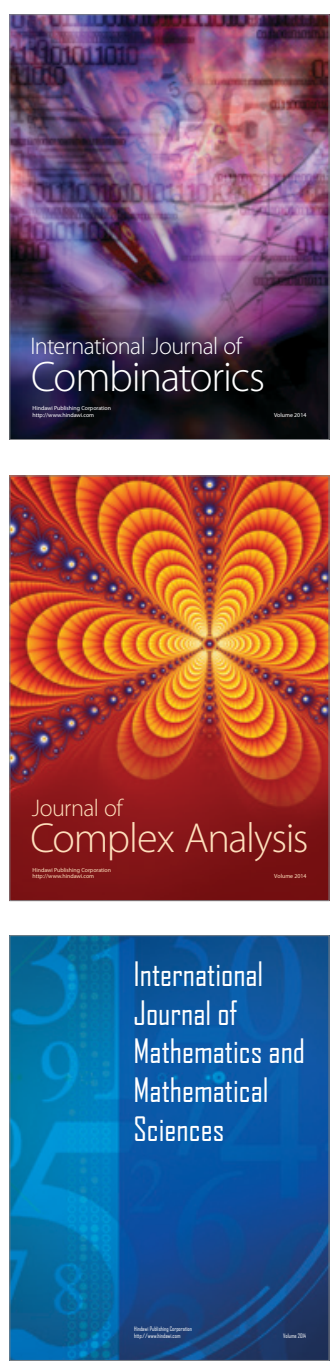
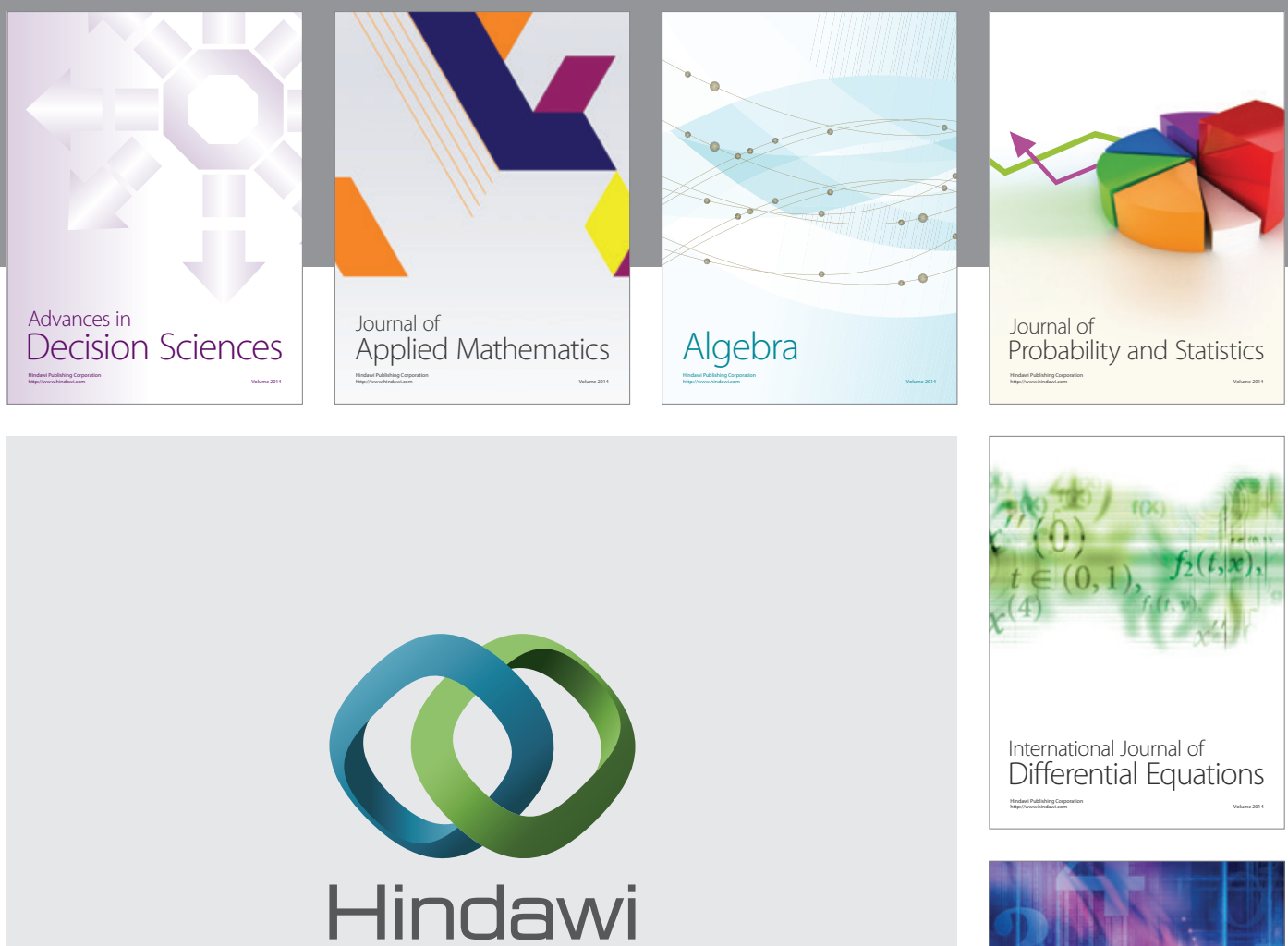

Submit your manuscripts at http://www.hindawi.com
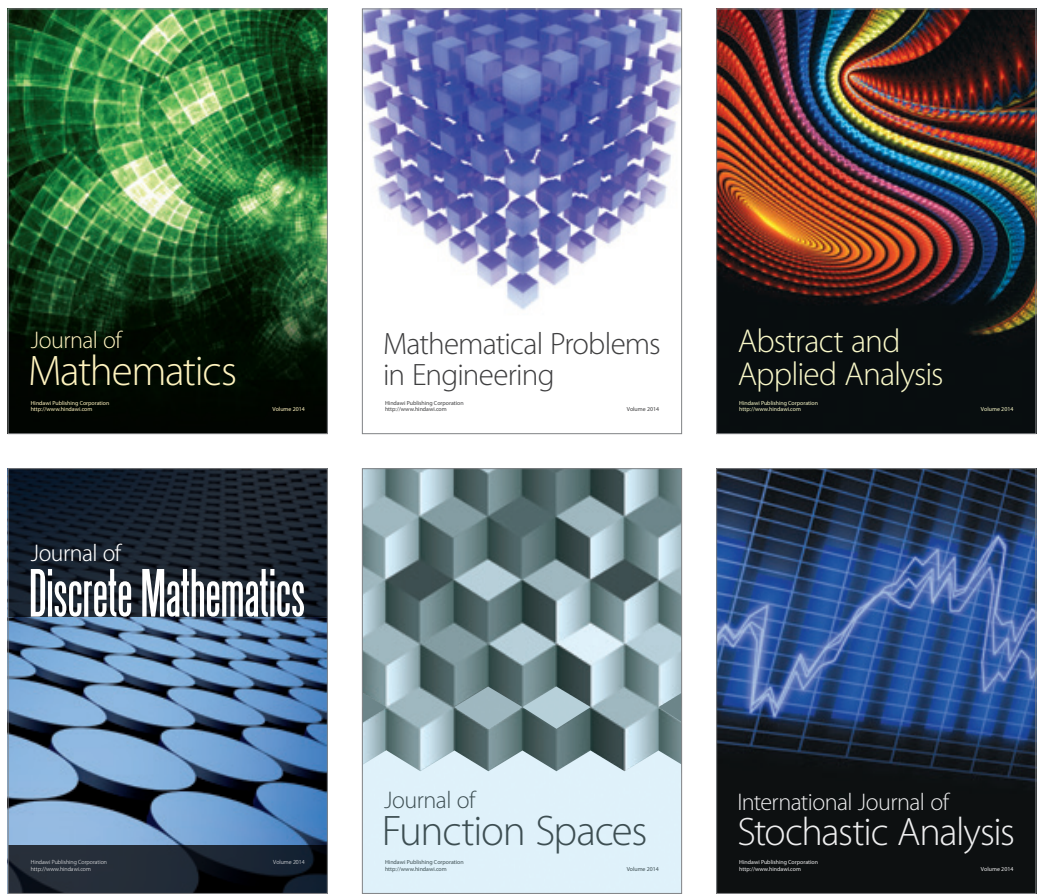

Journal of

Function Spaces

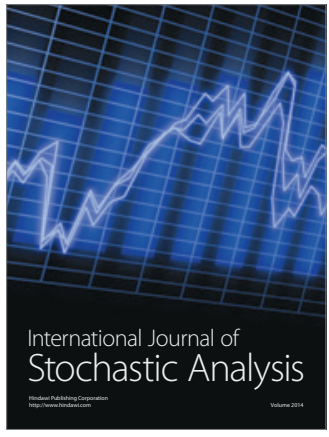

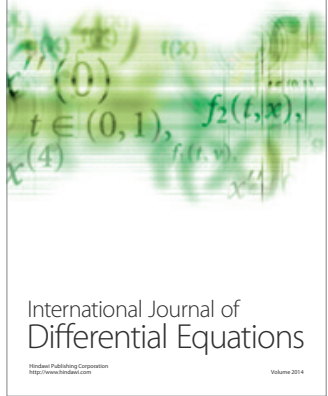
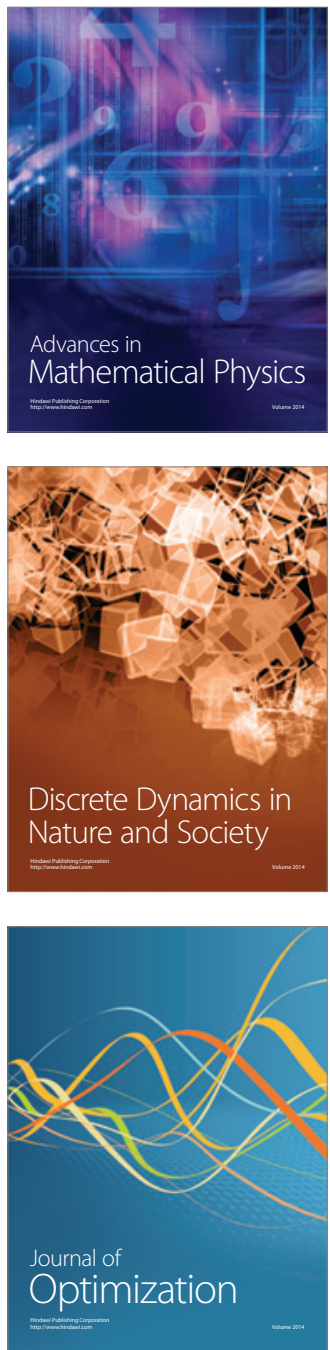